\title{
Moderne Probleme der Entwicklung des Versicherungswesens in der Sowjetunion
}

\author{
von L. I. Reitman*
}

Im Zusammenhang mit der Perestroika in der Sowjetunion, die in erster Linie das Finanz- und Versicherungswesen anbetrifft, entsteht gesetzmäßig das gegenseitige Interesse, sowohl in der UdSSR als auch im Ausland, an der Erforschung der internationalen Erfahrung auf dem Gebiet des Versicherungswesens, des Funktionierens des Versicherungsmarktes.

Erst jetzt beginnt in der Sowjetunion die Gestaltung des Versicherungsmarktes auf dem Gebiet der Innenversicherung. Bisher gab es das Staatsmonopol des Versicherungswesens, das der Gosstrach der UdSSR durchführte. Es schuf keine Voraussetzungen für die Existenz des Versicherungsmarktes; es war ein Markt mit einem Verkäufer und einem streng festgelegten Kreis der Käufer der "Versicherungsware".

Außerdem wurde die Rolle des Versicherungswesens als effektive Methode des Schadenersatzes für Naturkatastrophen lange Zeit unterschätzt. Dafür wurde die Abfindung der Natural- und Finanzressourcen aus dem all-unionischen Reservefonds bevorzugt. Es stellte Zweigministerien, Betriebe und Betriebsvereinigungen, die eine Naturkatastrophe erlebt hatten, zufrieden. Sie waren überzeugt, daß der Staat ihnen immer hilft, wenn die eigene Finanzreserve für den Schadenersatz nicht reicht. Als Ergebnis wußten wenige der Wirtschaftler überhaupt, was das Versicherungswesen bedeutet, seine Vorzüge und worin die unbedingte Notwendigkeit der Versicherung besonders bei der wirtschaftlichen Rechnungsführung und der Erwirtschaftung besteht.

Deshalb entstanden im Laufe der Umgestaltung des Versicherungswesens in der UdSSR drei Probleme:

1. Das Problem des neuen Sinns des Platzes und der Rolle der Versicherung im wirtschaftlichen Leben der Gesellschaft.

2. Das Problem des Versicherungswesens und der freiwilligen Versicherung und der optimalen Proportion zwischen diesen Organisationsformen des Versicherungswesens.

3. Das Problem der Demonopolisierung des Versicherungswesens in der UdSSR, der Gestaltung des Versicherungsmarktes und des Platzes des Gosstraches der UdSSR auf diesem Markt.

\footnotetext{
* Professor, Moskauer Finanzinstitut.
} 
Diese drei Probleme umfassen die ganze Gesamtheit der modernen Probleme der Versicherungswesensentfaltung natürlich nicht. Aber wir möchten unsere Aufmerksamkeit nur ihnen, als aktuellsten, schenken. Jedoch ist der Autor bereit, Fragen der Leser zu beantworten, die das Versicherungswesen der UdSSR betreffen.

Betrachten wir die drei bezeichneten Probleme:

1. Der enge Kreis der Fachleute, und zwar Wissenschaftler und praktische Mitarbeiter der Versicherungsanstalten, war mit den Fragen der Theorie des Versicherungswesens in der UdSSR, die das ökonomische Wesen, die Bestimmung, den Platz und die Rolle in der Sicherstellung des Versicherungsschutzes der gesellschaftlichen Produktion und der Bedürfnisse des Volkes erfassen, beschäftigt. Die Umgestaltung des ökonomischen Lebens steigerte das Interesse an der Versicherung in den breiten Schichten des Volkes, besonders bei den Ökonomen und Massenmedien. Die Erörterung der Versicherungsfragen in der Presse ist Mode; fast jeden Tag spricht jemand über das Versicherungswesen in den Zeitungen oder im Fernsehen. Dabei erscheinen sowohl sachliche Überlegungen zu diesem Thema, als auch unkompetente und sogar unwissende Äußerungen.

Ein Verfasser zum Beispiel nannte seinen Artikel über das Versicherungswesen in der Zeitung "Die Wirtschaft und das Leben" (das Organ der Geschäftsleute in der UdSSR) “den Bärendienst". Nach Auffassung des Verfassers besteht der Bärendienst darin, daß der Gosstrach Milliarden Rubel akkumuliert und niemand weis, wie er sie nutzt. Es wäre sehr schön, schreibt der Autor, wenn die einzelnen Wirtschaften ohne Gosstrach einander Geldhilfe leisten, wann man sie braucht.

Die (schlechtinformierte) Menge der Versicherungsnehmer hat die "LieschenMüller-Meinung" über die Versicherung als eine Art von Unterstützungskasse, die auf Rechnung der bestimmten fixierten Beiträgen der Fonds das Geld beschafft.

Die Auseinandersetzung mit der Versicherung schuf bei gewissen Wirtschaftlern eine andere Tendenz: Organisicrung der ressortmäßigen Selbstversicherung. Einige Ressorts, wie das Ministerium des Verkehrswesens, beabsichtigten ohne Gosstrach den Schadenersatz mit Hilfe der eigenen Reservefonds durchzuführen. Aber auf Rat der Spezialisten liess es seine Idee fallen. Die bezeichneten Reservefonds reichen nicht für den Schadenersatz im Katastrophenfall, wie z. B. in Baschkirien: Wegen der Explosion der Erdgasleitung im Jahre 1989 verbrannten zwei Passagierzüge, Schaden cirka eine Milliarde Rubel. Durch die Verbreitung der negativen Einstellung zur Versicherung und das naive Streben, sie durch Zusammenhilfe und Selbstversicherung zu ersetzen, mußten Spezialisten auf dem Gebiet der Versicherung den Versicherungsnehmern die spezifische Besonderheit des Versicherungswesens erklären, insbesondere folgende kennzeichnende Züge der Versicherung:

Während der Versicherung wird die Umverteilung des Schadenbeitrages zwischen den Teilnehmern der Versicherungsfonds durchgeführt. Die Versicherungsbeträge sind eine mathematische Wahrscheinlichkeit des Schadens, der auf jeden Versicherungsnehmer kommt. Je breiter dabei der Versicherungsnehmerkreis ist, desto billiger sind die Versicherungsbeiträge, weil man den Schadenbeitrag auf die größere Zahl der Mitglieder verteilt. Die Versicherungsbeiträge besorgen die Umverteilung des Schadens sowohl im Maßstab des Territoriums, als auch im Maßstab der Zeit. Findet im laufenden Jahr keine 
Naturkatastrophe statt, wird ein besonderer Teil des Versicherungsfonds als Reserve angerechnet, die man für den künftigen Schadenersatz braucht, wenn die laufenden Beiträge für die Versicherungsentschädigung nicht reichen könnten. Im Versicherungsfonds gibt es also immer die notwendigen Mittel für die Versicherungsentschädigung im Fall der (Natur-)Katastrophe. Darin ist sein Hauptunterschied zur Unterstützungskasse.

Es gibt noch eine Eigenheit der Versicherung, die nicht jeder versteht: die solidare Umverteilung der Schadensumme zwischen den Versicherungsnehmern. Weil eine Naturkatastrophe normalerweise nicht jeden, sondern nur einigen Versicherungsnehmern Schaden bringt, wird der Schadenersatz von den ungeschädigten Wirtschaften für Wirtschaften, die zum Schaden gekommen sind, als Umverteilung der Versicherungsbeiträge durchgeführt. Darin besteht die Eigenheit der Versicherung.

Weiter wird die eigenheitliche verschlossene Umverteilung des Schadens nur bei der großen Zahl der Teilnehmer möglich. Mit anderen Worten, die Versicherung fördert die Bildung zentralisierter Versicherungsfonds. In diesem Prozeß der Zentralisierung des Versicherungsfondsmittels kann man die Rückversicherung als effektive Methode der Vertiefung des Mechanismus der Schadenumverteilung anwenden.

Und schließlich ist der Versicherungsfonds rückgekoppelt für die Gesamtheit der Versicherungsnehmer, die daran teilgenommen haben, außer den Unkosten des Versicherungsgebers. Diese letzte Eigenheit der Versicherung ist ein Offenbarung für viele Wirtschafter, die Versicherungsbeiträge und Steuern oft gleichsetzen.

Zur Zeit ist ein Gesetzesentwurf über die Grundlagen des Versicherungswesens in Vorbereitung, in dem ein Versuch gemacht wird, die Grundsätze eines gegenseitig verbundenen Systems aller Arten und Formen der Versicherung in der UdSSR zu formulieren. Dieses Gesetz verdient die Aufmerksamkeit breiter Gesellschaftskreise und könnte ein Fundament für ein richtiges Verstehen des Versicherungswesens, seines Platz und seiner Rolle in der Wirtschaft des Landes werden.

2. Die Umgestaltung des wirtschaftlichen Mechanismus der UdSSR erregte die negative Haltung der Wirtschafter und der Presse zum Versicherungszwang als ein Diktat, eine Steuer, eine Verletzung der Unabhängigkeit der Betriebe und der Bürger.

Das Problem des Versicherungszwangs und der freiwilligen Versicherung ist mit der Versicherungskultur der Wirtschafter und der Bevölkerung verbunden. Unter Versicherungskultur verstehen wir die vom Versicherungsnehmer akzeptierte Notwendigkeit des Schutzes seiner materiellen Interessen. Die Jahrhundertalte Geschichte der Versicherung in den europäischen und anderen hochentwickelten Ländern formierte bei den Versicherungsnehmern das stabile Bedürfnis nach der Versicherung ihrer materiellen Interessen, das heißt, ein hohes Niveau der Versicherungskultur: eine Versicherungspolice, ein Merkmal des Wohlstandes. In der Sowjetunion gibt es eine andere Situation. Der enge Rahmen des Versicherungsbereiches und das Fehlen insbesondere der Versicherung des Produktionsvermögens (außer landwirtschaftlichem Vermögen) im Laufe von fast 70 Jahren hat eine Erwartungshaltung bei vielen Wirtschaftern hervorgerufen, daß der außerordentliche Schaden vom Staat automatisch ersetzt wurde, sogar auf Rechnung des Staatshaushaltes. Wenn beim Übergang zur wirtschaftlichen Rechnungsführung der Betriebe die Hauptquelle des außerodentlichen Schadenersatzes die Versicherung wird, streben diese Wirtschafter danach, die Versicherung zu vermeiden und glauben, mit Hilfe des Staates die Brand- und Havarieverluste ersetzen zu können. 
Wegen des niedrigen Niveaus der Versicherungskultur erscheint die Notwendigkeit des Versicherungszwangs für bestimmte Vermögensteile deshalb unumgänglich Es geht darum, aus allen Objekten des Eigentums, sowohl des gesellschaftlichen Eigentums, als auch des kolchos-kooperativen Eigentums, das besonders betroffene Vermögen zu wählen, dessen Untergang oder Beschädigung die Interessen nicht nur der Versicherungsnehmer, sondern der ganzen Gesellschaft betrifft. Zu diesem prioritären Vermögen gehört in erster Linie die Ernte der landwirtschaftlichen Kulturen und die Ergebnisse der Viehzucht in Kolchosen und Sowchosen. In der UdSSR ist die Ernte der landwirtschaftlichen Kulturen gegen alles (inklusive der Dürre) versichert, im Gegensatz zu den meisten anderen Ländern. Die Unversehrtheit und unablässiger Wuchs der Ernte ist ein Kern der Lösung des lebensnotwendigen Lebensmittelprogramms in unserem Land. Beim Untergang der Ernte, einer Viehseuche oder bei Naturkatastrophen ist die Unterstützung der Landwirtschaftsbetriebe und die Erforschung zusätzlicher Quellen von Lebensmittelressourcen eine Notwendigkeit. Der Staat wird in bedeutendem Maße von der Entschädigung des erlittenen Verlustes befreit, wenn das Vermögen der Landwirtschaftsbetriebe versichert ist. Ausgehend davon, bestimmte das Gesetz die obligatorische Versicherung des Vermögens der Landwirtschaftsbetriebe.

Das Erdbeben, das im Dezember 1988 Armenien heimsuchte, bestätigte überzeugend die Rationalität des Versicherungszwangs in der Landwirtschaft. Der Verlust der Kolchosen und Sowchosen wurde dank der obligatorischen Versicherung im vollen Umfang entschädigt. Bei der freiwilligen Versicherung hätte die Versicherungsentschädigung nur $10 \%$ des Verlustes betragen, den Rest hätte der Staatshaushalt entschädigen müssen, der zur Zeit defizitär ist. Man kann auch mit Sicherheit annehmen, daß viele Landwirtschaftsbetriebe ihr Vermögen überhaupt nicht versichern würden.

Die Grund- und Umlauffonds der staatlichen Betriebe, die die Grundlage der wirtschaftlichen Produktion bilden, sind weitere vorrangige Objekte. Deshalb bespricht jetzt der Oberste Sowjet der UdSSR den Entwurf des Gesetzes über die obligatorische Versicherung der Betriebsvermögen.

Unter den Objekten in Privatbesitz sind die Wohnhäuser vorrangig. In der UdSSR muß man sie im Umfang von $40 \%$ ihrer Kosten obligatorisch versichern; die übrigen $60 \%$ sind die Sphäre der freiwilligen Versicherung. Die freiwillige Versicherung umfaßt auch weitere Objekte, die den Bürgern und Genossenschaften gehören. Besonders populär ist in der UdSSR die Versicherung des Hausrates, der Fahrzeuge und der Nutztiere, die den Bürgern gehören.

Die freiwillige persönliche Versicherung schließt Krankenversicherung, Lebensversicherung für Todesfall und Erlebensfall (gemischte Versicherung, Kinderversicherung und Hochzeitsversicherung) sowie die Versicherung der zusätzlichen Altersrente ein. Außerdem bestehen verschiedene Arten der Unfallversicherung, einschließlich der obligatorischen Versicherung der Fahrgäste. Die Personenversicherung ist in der UdSSR in bedeutendem Maße eine Ergänzung der staatlichen Sozialversicherung und sozialen Fürsorge. Die meisten Arbeiter und Angestellten bekommen doppelte Unterstützung im Krankheits- oder Todesfall: von der sozialen Fürsorge und der privaten Versicherung, wenn der Bürger versichert wurde.

Die Besonderheit der gegenwärtigen Etappe der Versicherungsentwicklung in der UdSSR besteht in der Demonopolisierung des Versicherungswesens und in der Bildung des Versicherungsmarktes im Inland. 
Obwohl bis 1988 das unbegrenzte Versicherungsmonopol von Gosstrach die UdSSR beherrschte, zeigt cine sorgfältige Analyse der sowjetischen Gesetzgebung, daß es kein Gesetz gibt, das dieses Monopol zwingend bestimmt. Dic Verfassung der UdSSR (Artikel 131) weist darauf hin, daß die Gestaltung der staatlichen Versicherung zur Kompetenz des Ministerrates gehört. Doch folgt daraus nicht, daß nur Gosstracht allein dic Versicherungseinrichtung sein kann.

Das staatliche Versicherungsmonopol wurde vom Gesetz über Genossenschaftswesen, das im Jahre 1988 angenommen wurde und die Durchführung der Genossenschaftsversicherung erlaubte, gebrochen. Es entstanden genossenschaftliche und Versicherungsaktiengesellschaften, die die Versicherung der Unternehmenstätigkeit (auf den Einkommensverlust, auf die Kreditnichtzahlung und auf die Produktionsnichtlieferung), die Versicherung der Arbeiter und Angestellten für den Krankhcitsfall und einige anderc Arten der Versicherung anbieten.

Doch in der Tätigkeit dieser Vcrsicherungsanstalten zeichnct sich einc negative Tendenz auf: sie zeigen sichtliche Abenteuerlichkeit im Auswahl der Versicherungsgegenstände, im Umfang der Versicherungsdeckung, in der Berechnung der Versicherungsprämie. Inflation und steigender Warenmangel können die Versicherung der Unternehmenstätigkeit, besonders die Versicherung auf Nichtzahlung, zu Benachteiligungen der Versicherungsanstalten führen. Dies ist schon in einigen Gebieten des Landes geschehen.

Im Zusammenhang damit entstand das Problem der staatlichen Versicherungsaufsicht. Es wurde der Entwurf eines Regierungsbeschlusses über die Versicherungsaufsicht vorbereitet, die zum unabhängigen Kontrollorgan werden muß.

Die Versicherungsaufsicht hat folgende Aufgaben:

- Ausgabe der Lizenzen für die Durchführung der Versicherungsoperationen und Entziehung der Lizenzen in bestimmten Fällen;

- die Bestätigung des Minimalmaßes des Statutfonds und des Reservefonds;

- die Bestätigung des Maßes des Versicherungszinses für jede Versicherungsart und die Kontrolle der Zinspolitik des Versicherungsgebers;

- die Kontrolle der Investitionstätigkeit des Versicherungsgebers;

- die Kontrolle der jährlichen Finanzergebnisse des Versicherungsgebers; Ausgabe von Empfehlungen für die Kräftigung seines Finanzzustandes.

Es scheint befremdlich, daß die Kontrolle der Zinspolitik eine der führenden Funktionen der Versicherungsaufsicht ist. Doch in unseren Bedingungen ist es für die Vorbeugung der Möglichkeit der lıaltlosen Überhöhung des Zinses, zunı Empfang des zusätzlichen Gewinns notwendig.

Im Zusammenhang mit der Entwicklung des Versicherungsmarktes entstand das Problem der Festlegung der Rolle von Gosstrach der UdSSR auf dicsem Markt. Ausgehend davon, daß Gosstrach eine mächtige Versicherungsanstalt mit Finanzressourcen im Umfang etwa 30 Milliarden Rubel ist, muß Gosstrach am Versichcrungsmarkt cine führende Lage cinnehmen. Sein Hauptzweck wird in der Entschädigung außerordentlicher Schäden bestehen, weil finanzschwache genosscnschaftliche und andcre Versicherungsanstalten nur lokalc Verluste entschädigen können.

Staatliche Vcrsicherung muß sowohl obligatorisch als auch freiwillig scin. Obligatorisch können vorrangigc Objekte versichert werden. Die übrigeı Arten der Vermögens- 
und Personenversicherung können freiwillig durchgeführt werden. Führenden Platz nimmt hier die Lebensversicherung und die Versicherung der zusätzlichen Rente, dic alljährlich Milliardenversichcrungslcistung für die Versichcrungsnchmer fördert.

Es ist bekannt, daß in vielen kapitalistischen Ländern mit einem entwickelten Versicherungsmarkt auch dic staatlichc Versicherung existiert. Die Notwendigkeit der staatlichen Versicherung folgt aus den staatlichen Interessen dieser Länder. Der Staat, zum Beispiel, nimmt auf sich die Versichcrung gegen Kricgsrisiko, die Versicherung der Stadtwirtschaft gegen Folgen von Bürgcrunruhen, die Versicherung der Renten der besonderen Sozialschichten and andere Arten der Versicherung.

Die staatliche Versicherungsaufsicht muss auch Gosstrach einschließen, so daß die staatliche Versicherung gleiche Bedingungen am Versicherungsmarkt wie private Versicherungsanstalten vorfindet.

Dic breitc Entwicklung dcs Vcrsicherungsmarktes ist ohne Rückversicherung unmöglich. Die Notwendigkeit der Rückversicherung folgt aus der Förderung der Konzentration des Versicherungsfonds, des Schaffens der notwendigen finanziellen Standfestigkeit der Versicherungsoperationen für jeden Versicherungsgeber am Versicherungsmarkt. Der Gosstrach der UdSSR als die mächtigste Versicherungsanstalt kann auch ein wichtiger Rückversicherer für genossenschaftliche, gegenseitige und Versicherungsaktiengesellschaften werden.

Die Gestaltung des Versicherungsmarktes in der UdSSR fordert die theoretische Definition des Begriffes "Versicherungsmarkt" (Sinns, Inhalts und Zwecks). Viele ausländische Autoren begrenzen sich auf die Begriffsbestimmung des Versicherungsmarktes als die Gesamtheit der Versicherungsgeber, Rückversicherungsgeber und der Sphäre, wo sie die Versicherungsdienste erweisen. Doch ist es offensichtlich, daß der Versicherungsmarkt viel breitere Sphären der Versicherungsbeziehungen umfaßt, die auch die Interessen des Käufers und des Verkäufers der Versicherungsdienstleistung in diesem eigenartigen Markt einschließt. Die Gesamtheit der Versicherungsinteressen beider Seiten führt zur optimalen Kombination der obligatorischen und freiwilligen Sicherstellung, zur Vielfältigkeit der Versicherungsarten, zum Spezifischen der Versicherungsdienstleistung und zur Behandlung des Versicherungsbeitrages. In der Literatur ist die Definition der Versicherungsware entweder als Bedürfnisse des Versicherungsnehmers zu der Versicherungsverteilung, oder als Versicherungsdeckung verbreitet. Doch das Spezifische der Versichcrungsdienstlcistung bestcht in ihrem doppelten Charakter. Der Versicherungsnehmer interessiert sich beim Kauf des Versicherungsscheines für die Versicherungsdeckung des cntsprechenden Risikos. Aber wenn im Laufe des nächsten Jahres oder im Laufe mehrerer Jahre kein Versicherungsfall eintritt, so wird auch keine Entschädigung ausbezahlt. Dann vcrwandclt sich im Bcwußtsein des Versicherungsnehmers der von ihm bezahlte Versicherungsbeitrag in Kosten des Risikos des möglichen Versicherungsfalls. Dadurch unterscheidet sich die Versicherungsware von ciner gcwöhnlichen Ware - von Produktion oder vom gcwöhnlichen Dienst, die der Käufer gleichzeitig verwendet. Der Versicherungsdienst, die Versicherungsware bilden cinc ständige Garantie der Versicherungsdeckung, aber diese Deckung kann als Entschädigung in Geldform der bestimmten Ergebnisse des möglichen Versicherungsfalls geschehen. Die Verbreitung dieser Bestimmungen unter den Versicherungsnehmern kann die Entwicklung des Vcrsichcrungsmarktes fördern. 